\title{
Development of a New One-Step Scheme for the Solution of Initial Value Problem (IVP) in Ordinary Differential Equations
}

\author{
Fadugba Sunday Emmanuel ${ }^{1, *}$, Falodun Bidemi Olumide ${ }^{2}$ \\ ${ }^{1}$ Department of Mathematics, Ekiti State University, Ado Ekiti, Nigeria \\ ${ }^{2}$ Department of Mathematics, University of Ilorin, Ilorin, Nigeria \\ Email address: \\ emmasfad2006@yahoo.com (F. S. Emmanuel) \\ ${ }^{*}$ Corresponding author
}

\section{To cite this article:}

Fadugba Sunday Emmanuel, Falodun Bidemi Olumide. Development of a New One-Step Scheme for the Solution of Initial Value Problem (IVP) in Ordinary Differential Equations. International Journal of Theoretical and Applied Mathematics. Vol. 3, No. 2, 2017 , pp. 58-63. doi: $10.11648 /$ j.ijtam.20170302.12

Received: October 27, 2016; Accepted: January 16, 2017; Published: February 9, 2017

\begin{abstract}
In this paper, a new one-step scheme was developed for the solution of initial value problems of first order in ordinary differential equations. In its development a combination of interpolating function and Taylor series were used. The method was used for the solution of initial value problems emanated from real life situations. The numerical results showed that the new scheme is consistent, robust and efficient.
\end{abstract}

Keywords: Interpolating Function, Initial Value Problem, One-Step Method, Ordinary Differential Equation, Taylor Series

\section{Introduction}

In the past years, a large number of methods suitable for solving ordinary differential equations have been proposed. A major impetus to developing numerical procedures was the invention of calculus by Newton and Leibnitz, as this led to accurate mathematical models for physical reality, such as Sciences, Engineering, Medicine and Business. These mathematical models cannot be usually solved explicitly and numerical method to obtain approximate solutions is needed.

The approach for the solution of initial value problems in ordinary differential equations based on numerical approximations were developed before the existence of programmable computers.

A numerical method is a complete and unambiguous set of procedures for the solution of a problem, together with computable error estimates. The study and implementation of such methods is the province of numerical analysis [12].

Development of numerical integrator for the solution of initial value problems in ordinary differential equations has attracted the attention of many researchers in recent years. There are numerous methods that produce numerical approximations to solution of initial value problem in ordinary differential equation such as Euler's method which was the oldest and simplest method originated by Leonhard Euler in 1768, Improved Euler method, Runge Kutta methods described by Carl Runge and Martin Kutta in 1895 and 1905 respectively.

There are many excellent literature and exhaustive texts on this subject that may be consulted, such as [1], [3], [4], [5], [6], [7], [8], [9], [11], [12] just to mention a few.

In this paper we consider the initial value problem of the form

$$
y^{\prime}=f(x, y), y(a)=y_{0}, x \in[a, b], y \in \mathbb{R}
$$

and develop an algorithm which can effectively solve initial value problems in ordinary differential equation. Many researchers have solved the problem in (1). However, if the solution to (1) posseses a singularity point, a numerical integration formulae will be more effective. In another development, [10] recently discussed one-step method of Euler-Maruyama type for the solution of stochastic differential equations using varying step sizes. [2] derived a continuous linear multistep method using Hermite polynomials as basis functions.

In this paper we develop a new accurate scheme for the 
solution of the initial value problems in ordinary equation. The rest of the paper is organized as follows: Section Two is the development of a new scheme. Section Three consists of some basic concepts vital to the development of the new scheme. Section Four consists of numerical experiment and discussion of results. Section Five concludes the paper.

\section{Development of the New Scheme}

We develop a new one-step scheme in solving an initial value problem in ordinary differential equations as follows. We intend to solve problem (1) with power series polynomial such that

$$
y(x)=\sum_{i=0}^{1} \alpha_{i} x^{i}+\alpha_{2} e^{-x}
$$

With integration interval of [a,b] in the form $a=x_{0}<$ $x_{1}<\cdots<x_{n}<x_{n+1}<\cdots<x_{N}=b$ with step size $h$ given by $h=x_{n+1}-x_{n}$ such that $n=0,1, \ldots, N-1$.

Expanding (2) above we obtain the interpolant of the form:

$$
F(x)=\alpha_{0}+\alpha_{1} x+\alpha_{2} e^{-x}
$$

where $\alpha_{0}, \alpha_{1}$ and $\alpha_{2}$ are real undetermined coefficients.

At $x=x_{n}$, (3) becomes

$$
F\left(x_{n}\right)=\alpha_{0}+\alpha_{1} x_{n}+\alpha_{2} e^{-x_{n}}
$$

Also,

$$
F\left(x_{n+1}\right)=\alpha_{0}+\alpha_{1} x_{n+1}+\alpha_{2} e^{-x_{n+1}}
$$
yields

Differentiating (4) and let $F^{\prime}\left(x_{n}\right)=f_{n}$ and $F^{\prime \prime}\left(x_{n}\right)=f_{n}^{\prime}$

$$
F^{\prime}\left(x_{n}\right)=\alpha_{1}-\alpha_{2} e^{-x_{n}}=f_{n}
$$

Similarly,

$$
F^{\prime \prime}\left(x_{n}\right)=\alpha_{2} e^{-x_{n}}=f_{n}^{\prime}
$$

Simplifying (6) and (7) above we obtain

$$
\alpha_{1}=f_{n}+f_{n}^{\prime}
$$

and

$$
\alpha_{2}=\frac{f_{n}^{\prime}}{e^{-(b+n h)}}
$$

Since

$$
x_{n+1}=b+(n+1) h
$$

and

$$
x_{n}=b+n h
$$

It worth mentioning in the present paper that $b$ varies which makes our derived scheme an avenue to solve any problem whose initial condition is not limited only to $y(0)=1$.

Subtracting (4) from (5) and using (8) and (9) with $b=0$ yields

$$
F\left(x_{n+1}\right)-F\left(x_{n}\right)=h f_{n}+\left[h+\left(e^{-h}-1\right)\right] f_{n}^{\prime}
$$

Using the fact that

$$
y_{n+1}-y_{n}=F\left(x_{n+1}\right)-F\left(x_{n}\right)
$$

Substituting (12) into (13) we obtain

$$
y_{n+1}-y_{n}=h f_{n}+\left[h+\left(e^{-h}-1\right)\right] f_{n}^{\prime}
$$

Equation (14) is the new one-step scheme.

\section{Some Basic Concepts [9]}

We consider the following basic concepts which are very vital to the development of the new scheme.

\subsection{Stability}

A numerical method is said to be stable if the difference between the numerical solution and the exact solution can be made as small as possible, that is if there exists two positive numbers $e_{0}$ and $K$ such that the following holds.

$$
\left\|y_{n}-y\left(x_{n}\right)\right\| \leq K\left\|e_{0}\right\|
$$

\subsection{Consistency}

A numerical method with an increment function $\varphi\left(x_{n} ; y, h\right)$ is said to be consistent with the initial value problem (1) under consideration, if

$$
\varphi\left(x_{n} ; y, h\right)=f(x, y)
$$

\subsection{Convergence}

A numerical method is said to be convergent if for all initial value problem satisfying the hypothesis of the Lipschitz condition given by

$$
\left|f(x, y)-f\left(x, y^{*}\right)\right| \leq L\left|y-y^{*}\right|
$$

where the Lipschitz constant $L$ is denoted by $L=\max \left|f_{y}^{\prime}(x, y)\right|$. The necessary and sufficient conditions for convergence are the stability and consistency.

\subsection{Round off Error}

This can be defined as the error due to computing device. They arise because it is possible to represent all real numbers exactly on a finite-state machine. It can be represented mathematically as

$$
R_{n+1}=y_{n+1}-p_{n+1}
$$

where $y_{n+1}$ is the approximate solution and $p_{n+1}$ is the computer output. The magnitude depends in the storage and the arithmetic operation adopted. In such cases, double precision are employed to guarantee an adequate approximation. 


\section{Numerical Experiment and Discussion of Results}

This section presents a comparative study of the new scheme and the theoretical solution with different step sizes.

\subsection{Numerical Experiment}

It is usually necessary to demonstrate the suitability and applicability of the newly developed scheme. In this course, we translated the algorithm of our scheme into MATLAB programming language and implemented with a problem emanated from real life situations. The performance of the method was checked by comparing its accuracy and efficiency. The efficiency was determined from the number of iterations counts and number of functions evaluations per step while the accuracy is determined by the size of the discretization error estimated from the difference between the exact solution and the numerical approximations.

\subsubsection{Application of the New Scheme to Real Life Problem}

Let us assume that a colony of 1000 bacteria are multiplying at the rate of $r=0.8$ per hour per individual (i.e an individual produces an average of 0.8 off spring every hour, see (Hahn B. D (1997)). How many bacteria are there after 1 hour? If we assume that the colony grows continuously and without restriction.

\section{Solution}

It is possible to model this growth with a differential equation of first order of the form:

$$
\frac{d N(t)}{d t}=r N(t)
$$

with initial condition:

$$
N(0)=1000
$$

Equations (19) and (20) connote the IVP given by

$$
\frac{d N(t)}{d t}=r N(t), N(0)=1000, r=0.8
$$

where $N(t)$ is the population size at time $t$. The exact solution to (21) is obtained as

$$
N(t)=1000 e^{0.8 t}
$$

Take $h=0.0125$ and $h=0.0100, t \in[0,1]$. The results generated from our scheme for $h=0.0125$ and $h=0.01$ are

\begin{tabular}{|c|c|c|c|}
\hline t-value & Exact-solution & Computed-solution & Error \\
\hline 0.0125000 & 1010.050167084167900000 & 1010.049792316084100000 & $3.747681 \mathrm{e}-004$ \\
\hline 0.0250000 & 1020.201340026755800000 & 1020.200582957764600000 & $7.570690 \mathrm{e}-004$ \\
\hline 0.0375000 & 1030.454533953516800000 & 1030.453386937238100000 & $1.147016 \mathrm{e}-003$ \\
\hline 0.0500000 & 1040.810774192388200000 & 1040.809229467362700000 & $1.544725 \mathrm{e}-003$ \\
\hline 0.0625000 & 1051.271096376024200000 & 1051.269146064173400000 & $1.950312 \mathrm{e}-003$ \\
\hline 0.0750000 & 1061.836546545359600000 & 1061.834182650425400000 & $2.363895 \mathrm{e}-003$ \\
\hline 0.0875000 & 1072.508181254216700000 & 1072.505395660181200000 & $2.785594 \mathrm{e}-003$ \\
\hline 0.1125000 & 1094.174283705210400000 & 1094.170629877864900000 & $3.653827 \mathrm{e}-003$ \\
\hline 0.1250000 & 1105.170918075647700000 & 1105.166817466496500000 & $4.100609 \mathrm{e}-003$ \\
\hline 0.1375000 & 1116.278070458871300000 & 1116.273514456662300000 & $4.556002 \mathrm{e}-003$ \\
\hline 0.1500000 & 1127.496851579375700000 & 1127.491831444896900000 & $5.020134 \mathrm{e}-003$ \\
\hline 0.1625000 & 1138.828383324621900000 & 1138.822890188999500000 & $5.493136 \mathrm{e}-003$ \\
\hline 0.1750000 & 1150.273798857227300000 & 1150.267823720201700000 & $5.975137 \mathrm{e}-003$ \\
\hline 0.1875000 & 1161.834242728283000000 & 1161.827776456463700000 & $6.466272 \mathrm{e}-003$ \\
\hline 0.2250000 & 1197.217363121810200000 & 1197.209367287323600000 & $7.995834 \mathrm{e}-003$ \\
\hline 0.2375000 & 1209.249597657251600000 & 1209.241072787431700000 & $8.524870 \mathrm{e}-003$ \\
\hline 0.2500000 & 1221.402758160169900000 & 1221.393694429024000000 & $9.063731 \mathrm{e}-003$ \\
\hline 0.2625000 & 1233.678059956743500000 & 1233.668447394210500000 & $9.612563 \mathrm{e}-003$ \\
\hline 0.2750000 & 1246.076730587381000000 & 1246.066559077428300000 & $1.017151 \mathrm{e}-002$ \\
\hline 0.2875000 & 1258.600009929478100000 & 1258.589269208173800000 & $1.074072 \mathrm{e}-002$ \\
\hline 0.3000000 & 1271.249150321404800000 & 1271.237829974968000000 & $1.132035 \mathrm{e}-002$ \\
\hline 0.3125000 & 1284.025416687741700000 & 1284.013506150565900000 & $1.191054 \mathrm{e}-002$ \\
\hline 0.3250000 & 1296.930086665772000000 & 1296.917575218426000000 & $1.251145 \mathrm{e}-002$ \\
\hline 0.3375000 & 1309.964450733247500000 & 1309.951327500450600000 & $1.312323 \mathrm{e}-002$ \\
\hline 0.3500000 & 1323.129812337437000000 & 1323.116066286008800000 & $1.374605 \mathrm{e}-002$ \\
\hline 0.3625000 & 1336.427488025472300000 & 1336.413107962257300000 & $1.438006 \mathrm{e}-002$ \\
\hline 0.3750000 & 1349.858807576003100000 & 1349.843782145770500000 & $1.502543 \mathrm{e}-002$ \\
\hline 0.3875000 & 1363.425114132178100000 & 1363.409431815493000000 & $1.568232 \mathrm{e}-002$ \\
\hline 0.4000000 & 1377.127764335957400000 & 1377.111413447029000000 & $1.635089 \mathrm{e}-002$ \\
\hline 0.4375000 & 1419.067548593257500000 & 1419.049120176418500000 & $1.842842 \mathrm{e}-002$ \\
\hline 0.4500000 & 1433.329414560340600000 & 1433.310269120513300000 & $1.914544 \mathrm{e}-002$ \\
\hline
\end{tabular}
shown in Tables 1 and 2 below respectively.

\subsubsection{Table of Results}

Table 1. Comparative Analyses of the Results of the New Scheme with Theoretical Solution for $h=0.0125$. 


\begin{tabular}{|c|c|c|c|}
\hline t-value & Exact-solution & Computed-solution & Error \\
\hline 0.4625000 & 1447.734614663324700000 & 1447.714739649685000000 & $1.987501 \mathrm{e}-002$ \\
\hline 0.4750000 & 1462.284589434224900000 & 1462.263972116098100000 & $2.061732 \mathrm{e}-002$ \\
\hline 0.4875000 & 1476.980793882643000000 & 1476.959421347157100000 & $2.137254 \mathrm{e}-002$ \\
\hline 0.5000000 & 1491.824697641270600000 & 1491.802556790979700000 & $2.214085 \mathrm{e}-002$ \\
\hline 0.5125000 & 1506.817785112853700000 & 1506.794862663332400000 & $2.292245 \mathrm{e}-002$ \\
\hline 0.5250000 & 1521.961555618633800000 & 1521.937838096041300000 & $2.371752 \mathrm{e}-002$ \\
\hline 0.5375000 & 1537.257523548281600000 & 1537.232997286896800000 & $2.452626 \mathrm{e}-002$ \\
\hline 0.5500000 & 1552.707218511335900000 & 1552.681869651061600000 & $2.534886 \mathrm{e}-002$ \\
\hline 0.5625000 & 1568.312185490168800000 & 1568.285999974004000000 & $2.618552 \mathrm{e}-002$ \\
\hline 0.5750000 & 1584.073984994481600000 & 1584.046948565965100000 & $2.703643 e-002$ \\
\hline 0.5875000 & 1599.994193217360400000 & 1599.966291417979800000 & $2.790180 \mathrm{e}-002$ \\
\hline 0.6000000 & 1616.074402192893300000 & 1616.045620359466100000 & $2.878183 \mathrm{e}-002$ \\
\hline 0.6125000 & 1632.316219955378800000 & 1632.286543217396200000 & $2.967674 \mathrm{e}-002$ \\
\hline 0.6250000 & 1648.721270700127700000 & 1648.690683977070000000 & $3.058672 \mathrm{e}-002$ \\
\hline 0.6375000 & 1665.2911949458866000000 & 1665.259682944502200000 & $3.151200 \mathrm{e}-002$ \\
\hline 0.6500000 & 1682.027649698885900000 & 1681.995196910442500000 & $3.245279 \mathrm{e}-002$ \\
\hline 0.6625000 & 1698.932308618550200000 & 1698.898899316043500000 & $3.340930 \mathrm{e}-002$ \\
\hline 0.6750000 & 1716.006862184857900000 & 1715.972480420193700000 & $3.438176 \mathrm{e}-002$ \\
\hline 0.6875000 & 1733.253017867394600000 & 1733.217647468532300000 & $3.537040 \mathrm{e}-002$ \\
\hline 0.7000000 & 1750.672500296100200000 & 1750.636124864163100000 & $3.637543 \mathrm{e}-002$ \\
\hline 0.7125000 & 1768.267051433734400000 & 1768.229654340082300000 & $3.739709 \mathrm{e}-002$ \\
\hline 0.7250000 & 1786.038430750072600000 & 1785.999995133341400000 & $3.843562 \mathrm{e}-002$ \\
\hline 0.7375000 & 1803.988415397856000000 & 1803.948924160958800000 & $3.949124 \mathrm{e}-002$ \\
\hline 0.7500000 & 1822.118800390508100000 & 1822.078236197599800000 & $4.056419 \mathrm{e}-002$ \\
\hline 0.7625000 & 1840.431398781636300000 & 1840.389744055042700000 & $4.165473 \mathrm{e}-002$ \\
\hline 0.7750000 & 1858.928041846340900000 & 1858.885278763447100000 & $4.276308 \mathrm{e}-002$ \\
\hline 0.7875000 & 1877.610579264342100000 & 1877.566689754445900000 & $4.388951 \mathrm{e}-002$ \\
\hline 0.8000000 & 1896.480879304950100000 & 1896.435845046075400000 & $4.503426 \mathrm{e}-002$ \\
\hline 0.8125000 & 1915.540829013894800000 & 1915.494631429566000000 & $4.619758 \mathrm{e}-002$ \\
\hline 0.8250000 & 1934.792334402030100000 & 1934.744954658007100000 & $4.737974 \mathrm{e}-002$ \\
\hline 0.8375000 & 1954.237320635938000000 & 1954.188739636911600000 & $4.858100 \mathrm{e}-002$ \\
\hline 0.8500000 & 1973.877732230446100000 & 1973.827930616692600000 & $4.980161 \mathrm{e}-002$ \\
\hline 0.8625000 & 1993.715533243080700000 & 1993.664491387076400000 & $5.104186 \mathrm{e}-002$ \\
\hline 0.8750000 & 2013.752707470474900000 & 2013.700405473468000000 & $5.230200 \mathrm{e}-002$ \\
\hline 0.8875000 & 2033.991258646748700000 & 2033.937676335290700000 & $5.358231 \mathrm{e}-002$ \\
\hline 0.9000000 & 2054.433210643886000000 & 2054.378327566319100000 & $5.488308 \mathrm{e}-002$ \\
\hline 0.9125000 & 2075.080607674120500000 & 2075.024403097024800000 & $5.620458 \mathrm{e}-002$ \\
\hline 0.9250000 & 2095.935514494362500000 & 2095.877967398956600000 & $5.754710 \mathrm{e}-002$ \\
\hline 0.9375000 & 2117.000016612672400000 & 2116.941105691172700000 & $5.891092 \mathrm{e}-002$ \\
\hline 0.9500000 & 2138.276220496816200000 & 2138.215924148750700000 & $6.029635 \mathrm{e}-002$ \\
\hline 0.9625000 & 2159.766253784912400000 & 2159.704550113389400000 & $6.170367 \mathrm{e}-002$ \\
\hline 0.9750000 & 2181.472265498198800000 & 2181.409132306130700000 & $6.313319 \mathrm{e}-002$ \\
\hline 0.9875000 & 2203.396426255934300000 & 2203.331841042216600000 & $6.458521 \mathrm{e}-002$ \\
\hline 1.0000000 & 2225.540928492464900000 & 2225.474868448106000000 & $6.606004 \mathrm{e}-002$ \\
\hline
\end{tabular}

Table 2. Comparative Analyses of the Results of the New Scheme and Theoretical Solution for $h=0.01$.

\begin{tabular}{|c|c|c|c|}
\hline t-value & Exact-solution & Computed-solution & Error \\
\hline 0.0100000 & 1008.032085504273500000 & 1008.031893599467600000 & $1.919048 \mathrm{e}-04$ \\
\hline 0.0200000 & 1016.128685406094900000 & 1016.128298513728300000 & $3.868924 \mathrm{e}-04$ \\
\hline 0.0300000 & 1024.290317890621500000 & 1024.289732890798600000 & $5.849998 \mathrm{e}-04$ \\
\hline 0.0400000 & 1032.517505305118200000 & 1032.516719040404500000 & $7.862647 \mathrm{e}-04$ \\
\hline 0.0500000 & 1040.810774192388200000 & 1040.809783467408600000 & $9.907250 \mathrm{e}-04$ \\
\hline 0.0600000 & 1049.170655324470500000 & 1049.169456905503700000 & $1.198419 \mathrm{e}-03$ \\
\hline 0.0700000 & 1057.597683736611300000 & 1057.596274351180000000 & $1.409385 \mathrm{e}-03$ \\
\hline 0.0800000 & 1066.092398761505100000 & 1066.090775097962000000 & $1.623664 \mathrm{e}-03$ \\
\hline 0.0900000 & 1074.655344063813600000 & 1074.653502770922800000 & $1.841293 \mathrm{e}-03$ \\
\hline 0.1000000 & 1083.287067674958700000 & 1083.285005361474100000 & $2.062313 \mathrm{e}-03$ \\
\hline 0.1100000 & 1091.988122028197500000 & 1091.985835262436000000 & $2.286766 \mathrm{e}-03$ \\
\hline 0.1200000 & 1100.759063993978800000 & 1100.756549303389600000 & $2.514691 \mathrm{e}-03$ \\
\hline 0.1300000 & 1109.600454915582500000 & 1109.597708786311600000 & $2.746129 \mathrm{e}-03$ \\
\hline 0.1400000 & 1118.512860645045300000 & 1118.509879521496300000 & $2.981124 \mathrm{e}-03$ \\
\hline 0.1500000 & 1127.496851579375700000 & 1127.493631863766300000 & $3.219716 \mathrm{e}-03$ \\
\hline 0.1600000 & 1136.553002697060300000 & 1136.549540748973200000 & $3.461948 \mathrm{e}-03$ \\
\hline 0.1700000 & 1145.681893594861800000 & 1145.678185730792600000 & $3.707864 \mathrm{e}-03$ \\
\hline 0.1800000 & 1154.884108524913700000 & 1154.880151017813400000 & $3.957507 \mathrm{e}-03$ \\
\hline 0.1900000 & 1164.160236432112500000 & 1164.156025510925500000 & 4.210921e-03 \\
\hline
\end{tabular}




\begin{tabular}{|c|c|c|c|}
\hline t-value & Exact-solution & Computed-solution & Error \\
\hline 0.2000000 & 1173.510870991810200000 & 1173.506402841008400000 & $4.468151 \mathrm{e}-03$ \\
\hline 0.2100000 & 1182.936610647811000000 & 1182.931881406921300000 & $4.729241 \mathrm{e}-03$ \\
\hline 0.2200000 & 1192.438058650669500000 & 1192.433064413799700000 & $4.994237 \mathrm{e}-03$ \\
\hline 0.2300000 & 1202.015823096301600000 & 1202.010559911658400000 & $5.263185 \mathrm{e}-03$ \\
\hline 0.2400000 & 1211.670516964900800000 & 1211.664980834305400000 & $5.536131 \mathrm{e}-03$ \\
\hline 0.2500000 & 1221.402758160169900000 & 1221.396945038567500000 & $5.813122 \mathrm{e}-03$ \\
\hline 0.2600000 & 1231.213169548867700000 & 1231.207075343832200000 & $6.094205 \mathrm{e}-03$ \\
\hline 0.2700000 & 1241.102379000671800000 & 1241.095999571905600000 & $6.379429 \mathrm{e}-03$ \\
\hline 0.2800000 & 1251.071019428362400000 & 1251.064350587192200000 & $6.668841 \mathrm{e}-03$ \\
\hline 0.2900000 & 1261.119728828329500000 & 1261.112766337195600000 & $6.962491 \mathrm{e}-03$ \\
\hline 0.3000000 & 1271.249150321404800000 & 1271.241889893346100000 & $7.260428 \mathrm{e}-03$ \\
\hline 0.3100000 & 1281.459932194021300000 & 1281.452369492155400000 & $7.562702 \mathrm{e}-03$ \\
\hline 0.3200000 & 1291.752727939704100000 & 1291.744858576702000000 & $7.869363 \mathrm{e}-03$ \\
\hline 0.3300000 & 1302.128196300894400000 & 1302.120015838449300000 & $8.180462 \mathrm{e}-03$ \\
\hline 0.3400000 & 1312.587001311108500000 & 1312.578505259400800000 & $8.496052 \mathrm{e}-03$ \\
\hline 0.3500000 & 1323.129812337437000000 & 1323.120996154592600000 & $8.816183 \mathrm{e}-03$ \\
\hline 0.3600000 & 1333.757304123384500000 & 1333.748163214927900000 & $9.140908 \mathrm{e}-03$ \\
\hline 0.3700000 & 1344.470156832052900000 & 1344.460686550355400000 & $9.470282 \mathrm{e}-03$ \\
\hline 0.3800000 & 1355.269056089671900000 & 1355.259251733394900000 & $9.804356 \mathrm{e}-03$ \\
\hline 0.3900000 & 1366.154693029480100000 & 1366.144549843011600000 & $1.014319 \mathrm{e}-02$ \\
\hline 0.4000000 & 1377.127764335957400000 & 1377.117277508843200000 & $1.048683 \mathrm{e}-02$ \\
\hline 0.4100000 & 1388.188972289412500000 & 1388.178136955782700000 & $1.083533 \mathrm{e}-02$ \\
\hline 0.4200000 & 1399.339024810930800000 & 1399.327836048918700000 & $1.118876 \mathrm{e}-02$ \\
\hline 0.4300000 & 1410.578635507678700000 & 1410.567088338836800000 & $1.154717 \mathrm{e}-02$ \\
\hline 0.4400000 & 1421.908523718577500000 & 1421.896613107285200000 & $1.191061 \mathrm{e}-02$ \\
\hline 0.4500000 & 1433.329414560340600000 & 1433.317135413206400000 & $1.227915 \mathrm{e}-02$ \\
\hline 0.4600000 & 1444.842038973879400000 & 1444.829386139138900000 & $1.265283 \mathrm{e}-02$ \\
\hline 0.4700000 & 1456.447133771086600000 & 1456.434102037992500000 & $1.303173 \mathrm{e}-02$ \\
\hline 0.4800000 & 1468.145441681989700000 & 1468.132025780197900000 & $1.341590 \mathrm{e}-02$ \\
\hline 0.4900000 & 1479.937711402288600000 & 1479.923906001235100000 & $1.380540 \mathrm{e}-02$ \\
\hline 0.5000000 & 1491.824697641270600000 & 1491.810497349545400000 & $1.420029 \mathrm{e}-02$ \\
\hline 0.5100000 & 1503.807161170112100000 & 1503.792560534825700000 & $1.460064 \mathrm{e}-02$ \\
\hline 0.5200000 & 1515.885868870569100000 & 1515.870862376712500000 & $1.500649 \mathrm{e}-02$ \\
\hline 0.5300000 & 1528.061593784057300000 & 1528.046175853855400000 & $1.541793 \mathrm{e}-02$ \\
\hline 0.5400000 & 1540.335115161127300000 & 1540.319280153386900000 & $1.583501 \mathrm{e}-02$ \\
\hline 0.5500000 & 1552.707218511336400000 & 1552.690960720787400000 & $1.625779 \mathrm{e}-02$ \\
\hline 0.5600000 & 1565.178695653522000000 & 1565.162009310151900000 & $1.668634 \mathrm{e}-02$ \\
\hline 0.5700000 & 1577.750344766478300000 & 1577.733224034859900000 & $1.712073 \mathrm{e}-02$ \\
\hline 0.5800000 & 1590.422970440039800000 & 1590.405409418652900000 & $1.756102 \mathrm{e}-02$ \\
\hline 0.5900000 & 1603.197383726574600000 & 1603.179376447121100000 & $1.800728 \mathrm{e}-02$ \\
\hline 0.6000000 & 1616.074402192893800000 & 1616.055942619605000000 & $1.845957 \mathrm{e}-02$ \\
\hline 0.6100000 & 1629.054849972574900000 & 1629.035932001512900000 & $1.891797 \mathrm{e}-02$ \\
\hline 0.6200000 & 1642.139557818705700000 & 1642.120175277058500000 & $1.938254 \mathrm{e}-02$ \\
\hline 0.6300000 & 1655.329363157055700000 & 1655.309509802422800000 & $1.985335 \mathrm{e}-02$ \\
\hline 0.6400000 & 1668.625110139667400000 & 1668.604779659342700000 & $2.033048 \mathrm{e}-02$ \\
\hline 0.6500000 & 1682.027649698886800000 & 1682.006835709129700000 & $2.081399 \mathrm{e}-02$ \\
\hline 0.6600000 & 1695.537839601820500000 & 1695.516535647122700000 & $2.130395 \mathrm{e}-02$ \\
\hline 0.6700000 & 1709.156544505233600000 & 1709.134744057578500000 & $2.180045 \mathrm{e}-02$ \\
\hline 0.6800000 & 1722.884636010888000000 & 1722.862332469002000000 & $2.230354 \mathrm{e}-02$ \\
\hline 0.6900000 & 1736.722992721326400000 & 1736.700179409923500000 & $2.281331 \mathrm{e}-02$ \\
\hline 0.7000000 & 1750.672500296101600000 & 1750.649170465120300000 & $2.332983 \mathrm{e}-02$ \\
\hline 0.7100000 & 1764.734051508460200000 & 1764.710198332292300000 & $2.385318 \mathrm{e}-02$ \\
\hline 0.7200000 & 1778.908546302479000000 & 1778.884162879192700000 & $2.438342 \mathrm{e}-02$ \\
\hline 0.7300000 & 1793.196891850663400000 & 1793.171971201216400000 & $2.492065 \mathrm{e}-02$ \\
\hline 0.7400000 & 1807.600002612005300000 & 1807.574537679452100000 & $2.546493 \mathrm{e}-02$ \\
\hline 0.7500000 & 1822.118800390509700000 & 1822.092784039200100000 & $2.601635 \mathrm{e}-02$ \\
\hline 0.7600000 & 1836.754214394190500000 & 1836.727639408960600000 & $2.657499 \mathrm{e}-02$ \\
\hline 0.7700000 & 1851.507181294539100000 & 1851.480040379894700000 & $2.714091 \mathrm{e}-02$ \\
\hline 0.7800000 & 1866.378645286473300000 & 1866.350931065763900000 & $2.771422 \mathrm{e}-02$ \\
\hline 0.7900000 & 1881.369558148764100000 & 1881.341263163351400000 & $2.829499 \mathrm{e}-02$ \\
\hline 0.8000000 & 1896.480879304952200000 & 1896.451996013367500000 & $2.888329 \mathrm{e}-02$ \\
\hline 0.8100000 & 1911.713575884749200000 & 1911.684096661844700000 & $2.947922 \mathrm{e}-02$ \\
\hline 0.8200000 & 1927.068622785936000000 & 1927.038539922027000000 & $3.008286 \mathrm{e}-02$ \\
\hline 0.8300000 & 1942.547002736755100000 & 1942.516308436754100000 & $3.069430 \mathrm{e}-02$ \\
\hline 0.8400000 & 1958.149706358806700000 & 1958.118392741348800000 & $3.131362 \mathrm{e}-02$ \\
\hline 0.8500000 & 1973.877732230448600000 & 1973.845791327007600000 & $3.194090 \mathrm{e}-02$ \\
\hline 0.8600000 & 1989.732086950705000000 & 1989.699510704703000000 & $3.257625 \mathrm{e}-02$ \\
\hline
\end{tabular}




\begin{tabular}{llll}
\hline t-value & Exact-solution & Computed-solution & Error \\
\hline 0.8700000 & 2005.713785203689200000 & 2005.680565469595900000 & $3.321973 \mathrm{e}-02$ \\
0.8800000 & 2021.823849823545300000 & 2021.789978365967500000 & $3.387146 \mathrm{e}-02$ \\
0.8900000 & 2038.063311859907300000 & 2038.028780352672800000 & $3.453151 \mathrm{e}-02$ \\
0.9000000 & 2054.433210643888700000 & 2054.398010669118300000 & $3.519997 \mathrm{e}-02$ \\
0.9100000 & 2070.934593854599400000 & 2070.898716901770500000 & $3.587695 \mathrm{e}-02$ \\
0.9200000 & 2087.568517586197700000 & 2087.531955051199500000 & $3.656253 \mathrm{e}-02$ \\
0.9300000 & 2104.336046415478900000 & 2104.298789599659400000 & $3.725682 \mathrm{e}-02$ \\
0.9400000 & 2121.238253470012800000 & 2121.200293579212500000 & $3.795989 \mathrm{e}-02$ \\
0.9500000 & 2138.276220496819900000 & 2138.237548640399800000 & $3.867186 \mathrm{e}-02$ \\
0.9600000 & 2155.451037931604800000 & 2155.411645121465900000 & $3.939281 \mathrm{e}-02$ \\
0.9700000 & 2172.763804968546500000 & 2172.723682118135000000 & $4.012285 \mathrm{e}-02$ \\
0.9800000 & 2190.215629630644300000 & 2190.174767553951400000 & $4.086208 \mathrm{e}-02$ \\
0.9900000 & 2207.807628840633700000 & 2207.766018251183600000 & $4.161059 \mathrm{e}-02$ \\
1.0000000 & 2225.540928492468500000 & 2225.498560002297400000 & $4.236849 \mathrm{e}-02$ \\
\hline
\end{tabular}

\subsection{Discussion of Results}

The numerical experiment shows that the new scheme provides comparable results. The differences between our scheme and the theoretical solution are negligible from a practical point of view as we can see from Tables 1 and 2 above. Also we deduce that the smaller the step size, the more accurate is the new scheme. The numbers of bacteria present after 1 one hour for $h=0.0125$ and $h=0.01$ were obtained as 2225.474868448106000000 and 2225.498560002297400000 respectively.

\section{Conclusion}

In this paper, we have developed a new scheme for the solution of initial value problems in ordinary differential equations. The new scheme was used to obtain numerical solution to real life problem. The comparative analyses of the results were also presented. The numerical results of our scheme were compared favorably with the theoretical solution. Hence the new scheme is computational efficient, robust and easy to implement.

\section{References}

[1] Areo E. A. and Adeniyi R. B. (2014). Block implicit one-step method for the numerical integration of initial value problems in ordinary differential equations. International Journal of Mathematics and Statistics studies, 2 (3), 4-13.

[2] Aboiyar T., Luga T. and Ivorter B. V. (2015). Derivation of continuous linear multistep methods using Hermite polynomials as Basis functions. American Journal of Applied Mathematics and Statistics, 3 (6), 220-225.
[3] Boyce, W. E. and DiPrima, R. C. (2001). Elementary differential equation and boundary value problems. John Wiley and Sons.

[4] Collatz, L. (1960). Numerical treatment of differential equations. Springer Verlag Berlin.

[5] Erwin, K. (2003). Advanced engineering mathematics. Eighth Edition, Wiley Publisher.

[6] Fatunla S. O. (1980): "Numerical integrators for stiff and highly oscillatory differential equations", Mathematics of Computation 34, 373-390.

[7] Gilat, A. (2004). Matlab: An introduction with application. John Wiley and Sons. Gautschi W. (1961): "Numerical integration of ordinary differential equations based on trigonometric polynomials", NumerischeMathematik 3, 381397.

[8] Kayode S. J., Ganiyu A. A., and Ajiboye A. S. (2016). On one-step method of Euler-Maruyana type for solution of stochastic differential equations using varying stepsizes. Open Access Library Journal.

[9] Ogunrinde R. B. and Fadugba S. E. (2012). Development of a new scheme for the solution of initial value problems in ordinary differential equations. IOSR Journal of Mathematics, 2, 24-29.

[10] Wallace C. S and Gupta G. K. (1973). General Linear multistep methods to solve ordinary differential equations. The Australian Computer Journal, 5, 62-69.

[11] Ying T. Y., Zurni O. and Kamarun H. M. (2014). Modified exponential rational methods for the numerical solution of first order initial value problems. Sains Malaysiana, 43(12), 1951-1959.

[12] http://www.encyclopedia.com/computing/dictionariesthesauruses-pictures-and-press-releases/numerical-methods. 